\title{
A MAXWELL-BLOCH MODEL WITH DISCRETE SYMMETRIES FOR WAVE PROPAGATION IN NONLINEAR CRYSTALS: AN APPLICATION TO KDP
}

\author{
Christophe Besse ${ }^{1}$, Brigitte Bidégaray-Fesquet ${ }^{2}$, Antoine Bourgeade $^{3}$, \\ Pierre Degond ${ }^{1}$ AND Olivier SAut ${ }^{1,3}$
}

\begin{abstract}
This article presents the derivation of a semi-classical model of electromagnetic-wave propagation in a non centro-symmetric crystal. It consists of Maxwell's equations for the wave field coupled with a version of Bloch's equations which takes fully into account the discrete symmetry group of the crystal. The model is specialized in the case of a KDP crystal for which information about the dipolar moments at the Bloch level can be recovered from the macroscopic dispersion properties of the material.
\end{abstract}

Mathematics Subject Classification. 78A60, 81V80.

Received: October 28, 2003.

\section{INTRODUCTION}

The development of laser able to produce ultra-short pulses requires the elaboration of new models, adequate for describing non linear dispersion as well as polarization effects in the wave-crystal interaction. Indeed, for powerful laser sources, classical models like systems of nonlinear Schrödinger equations [3, 17] are no longer valid.

For such pulses, the hypothesis of monochromatic waves is no longer relevant. This requires a time-domain description rather than a spectral one as used in classical nonlinear optics $[2,3,10,14]$. Therefore, we shall consider Maxwell equations in the time domain and shall model the interaction of the electromagnetic field with the material medium by means of the Bloch equations.

Several models have been proposed for the polarization term which describes the response of the nonlinear material to the wave-field. Classically one writes the polarization as a development in powers of the electric field, the coefficients of this expansion are called the nonlinear susceptibilities. Experimental measures of the nonlinear susceptibilities as functions of the frequency of the incoming wave are scarce imprecise and of little use for a time-domain description. Analytical expressions of the nonlinear susceptibilities as functions of the dipolar matrices are given in [8]. We shall use this approach in the course of our analysis. Other theories can be found e.g. in [16], where the optical susceptibilities are related with covalent bonds in the crystal, or, in [24], where

\footnotetext{
Keywords and phrases. Nonlinear optics, optical susceptibility, harmonic generation, quantum description of light and matter, nonlinear optical crystals.

1 MIP, UMR 5640 (CNRS-UPS-INSA), Université Paul Sabatier, 118, route de Narbonne, 31062 Toulouse Cedex 4, France. e-mail: besse@mip.ups-tlse.fr, degond@mip.ups-tlse.fr, saut@mip.ups-tlse.fr

2 LMC, UMR 5523 (CNRS-UJF-INPG), B.P. 53, 38041 Grenoble Cedex 9, France. e-mail: Brigitte.Bidegaray@imag.fr

3 CEA/CESTA, B.P. 2, 33114 Le Barp, France. e-mail: Antoine.BOURGEADE@cea.fr
} 
the optical susceptibilities are related with the temperature. An example of the use of nonlinear susceptibilities in the time-domain can be found in [7]. In this approach, the Fourier transform of the polarization allows to write ordinary differential equations on the first orders of the polarization. These equations have to be solved for each time step. The quadratic susceptibility is taken independent of the frequency of the incoming wave.

Approaches based on susceptibilities can be classified into the so-called macroscopic models, because these coefficients describe the average response of the medium over large scales and a large assembly of atoms, and in particular, over distances larger than the wave-length. At variance, microscopic models describe the response of the medium on sub-wave-length scales. The Bloch model belongs to such microscopic models. The Bloch equation describes the quantum-mechanical response of the matter to the wave-field. This model accounts for the microscopic physics in a fairly accurate way, provided our knowledge of the elementary wave-matter interaction (namely the dipolar moment matrix) is known. We shall see that this point represents the true difficulty of our approach. The Bloch equation model was throughfully studied in the case when the dipolar moment vectors are parallel to the electric field (which is a model for isotropic media e.g. a gas) for two level atoms [26] or more [4]. We extend these approaches to an anisotropic nonlinear crystal with a discrete group of symmetry. In particular, with this model, we can investigate the interplay between anisotropy and nonlinearity.

In the Bloch model, the crystal is described locally by a density matrix whose diagonal elements represent the population and the off-diagonal elements, the quantum coherences of a set of atomic states. The density matrix evolves according to both the atomic and wave-Hamiltonians. While matter is treated quantum-mechanically, the wave-field is treated classically through Maxwell's equations, giving rise to the often-used terminology "semiclassical" model. The wave-Hamiltonian involves the matrix of dipolar moments, which describes how matter reacts to the wave-field at the microscopic level.

In the case of a crystal with symmetry, energy levels can be degenerate and the eigenstates span a linear representation of the symmetry group which must be decomposed into irreducible representations [1,6,22]. If $N$ energy levels are considered each being $d_{n}$-degenerate, the density matrix must be a square matrix of dimension $\left(\sum_{n=1}^{N} d_{n}\right)^{2}$ which is large. This is a first difficulty when dealing with crystals.

A discrete symmetry group often implies anisotropy. Because of anisotropy, each dipolar moment connecting two states $j$ and $k$ is a 3 -dimensional vector. However owing to symmetries, many entries of the dipolar matrices may be zero. The determination of the non-zero entries of the dipolar matrix and their values is the second difficulty of the problem.

These problems are far too complex to be solved in full generality, and we are going to restrict our analysis to one example, that of the KDP crystal. This crystal is of fundamental use in most high power laser experiments [12]. However, beyond this example, we believe our approach can be adapted to a large class of materials.

The symmetry group of the KDP crystal is known (the $\overline{4} 2 m$ crystallography group) as well as some of its macroscopic properties: its first order susceptibility has been measured and analytically fitted by Zernicke [25]; it has a tensorial form, and in its principal axis basis, its entries exhibit several resonances as functions of the wave frequency. Moreover the order of magnitude of the non-zero entries of its second order susceptibility is known $[9,11,13,15,23]$.

The first and second order susceptibilities can be related to the dipolar moment matrix through the method described in [8]. We shall use this relationship and, from the properties outlined above, try to extract some information about the dipolar moment matrix. This approach however requires a model for the structure of the KDP quantum states, which is not available.

Our approach will be to postulate the most simple quantum-state structure able to reproduce the symmetry and anisotropy properties of KDP together with the available information on the first and second order susceptibilities. This model has 5 states. The lower energy state is 3 -fold degenerate. The density matrix has therefore 10 independent complex entries and 5 real ones (owing to its hermitianity). The present paper describes how this simple model can be derived through to the procedures outlined here.

We end this section by outlining advantages and drawbacks of the present approach. In this model, nonlinear effects are not restricted to linear and quadratic ones as in the case of models based on optical susceptibilities. We shall also see that the nonlinearity introduced by the quantum expression of the polarization is bounded, 
at variance with optical susceptibility models. This feature results in a possible saturation of the nonlinearity which in the case of powerful laser sources may be important. However, our model involves heavy numerical computations. Thus it will be used either for short propagation distance or as a validation tool of simpler macroscopic models. For instance, it could be used to find dispersion relations for nonlinear Maxwell models.

The organization of this paper is as follows: Section 1 quickly exposes the physical context and the MaxwellBloch model.

As a first step in the determination of the dipolar moment matrix, we search in Section 2 a relation between some experimental evidences and the microscopic constants we need. Then, from this relation, we derive a set of conditions on the quantum structure of the crystal.

In Section 3, since this set of conditions is not sufficient to determine completely the unknowns, we study deeply the symmetries of the crystal and state their influence on the information we need. We can then develop a hierarchy of models.

In Section 4, we restrict ourselves to the study of the fundamental model, which is shown to be sufficient to solve our initial problem.

In Section 5, we give the full dipolar matrix moment computed in the case of the fundamental model.

In Section 6, we perform a numerical experiment with the fundamental model.

\section{Physical CONTEXT}

\subsection{Maxwell equations}

We consider plane waves. The incoming wave is represented by the pair $(\mathbf{E}, \mathbf{B})$, where $\mathbf{E}$ is the electric field and $\mathbf{B}$ the magnetic field. The fields $\mathbf{E}$ and $\mathbf{B}$ obey the Maxwell equations:

$$
\left\{\begin{array}{l}
\partial_{t} \mathbf{B}=-\operatorname{rot} \mathbf{E} \\
\partial_{t} \mathbf{E}=\frac{1}{\mu_{0}} \varepsilon^{-1} \operatorname{rot} \mathbf{B}-\varepsilon^{-1} \partial_{t} \mathbf{P} \\
\operatorname{div} \mathbf{B}=0 \\
\operatorname{div} \mathbf{D}=0
\end{array}\right.
$$

The interaction with the crystal is modeled by the polarization $\mathbf{P}$, which is obtained through the Bloch equations.

\subsection{Bloch equations}

We use a quantum model to represent the crystal. A quantum system is represented by a wave-function $\psi$. The evolution of $\psi$ is driven by the Schrödinger equation:

$$
i \hbar \partial_{t} \psi=\mathcal{H} \psi
$$

where the Hamiltonian $\mathcal{H}$ is classically decomposed in $\mathcal{H}=H_{0}+V, H_{0}$ being the free Hamiltonian and $V$ the potential resulting from the action of the electromagnetic field.

First, we introduce some notations. We make the assumption that the free Hamiltonian $H_{0}$ has $N$ distinct eigenvalues: $\mathcal{E}_{1}=\hbar \omega_{1}, \ldots, \mathcal{E}_{N}=\hbar \omega_{N}$. For convenience, we write $\omega_{n m}=\omega_{n}-\omega_{m}$.

We shall denote by $d_{n}$ the multiplicity of the eigenvalue $\mathcal{E}_{n}$. For each level $n$, we choose a basis of eigenstates $\left(\psi_{(n, 1)}, \ldots, \psi_{\left(n, d_{n}\right)}\right)$. For each pair of eigenstates $\left(\psi_{(l, r)}, \psi_{(m, s)}\right)$, we can compute $\boldsymbol{\mu}_{(l, r)(m, s)} \equiv\left\langle\psi_{(l, r)}, \mathbf{r} \psi_{(m, s)}\right\rangle$, where $\langle$,$\rangle is the canonical inner product and \mathbf{r}$ is the position operator. The matrix $\boldsymbol{\mu}$ is called the dipolar matrix. The dipolar matrix $\boldsymbol{\mu}$ is a $N \times N$ matrix whose coefficients are vectors in $\mathbb{C}^{3}$, thus we use a superscript to denote each coordinate of its elements. 
In the dipolar approximation, $V$ is given by

$$
V=-\boldsymbol{\mu} . \mathbf{E} .
$$

Now we introduce a statistical description of the system because a large number of atoms interacts with the wave. We introduce the density matrix $\rho$ described in [21] for instance. We just recall that the diagonal elements of the density matrix represent the population levels of the corresponding eigenspace of the free Hamiltonian, while the off-diagonal terms represent the coherences between these levels.

It is straightforward (e.g. see [8]) to show that the density matrix $\rho$ obeys the Bloch equations:

$$
\begin{aligned}
\partial_{t} \rho_{j k} & =-i\left(\omega_{j}-\omega_{k}\right) \rho_{j k}-\frac{i}{\hbar}[V, \rho]_{j k} \quad \text { (Bloch) } \\
& =-i \omega_{j k} \rho_{j k}+\frac{i}{\hbar}[\boldsymbol{\mu} . \mathbf{E}, \rho]_{j k}
\end{aligned}
$$

for all $1 \leq j, k \leq N$, where we recall $[A, B]=A B-B A$.

The two sets of equations (1) and (3) are related by the polarization $\mathbf{P}$ which is given by

$$
\mathbf{P}=\mathcal{N} \operatorname{tr}(\boldsymbol{\mu} \rho),
$$

where $\mathcal{N}$ is the density of molecules per unit volume.

Hence equations (1) and (3) form a closed system.

\section{EXPERIMENTAL RESULTS}

As we have seen, in order to use the Maxwell-Bloch model, we need to know several quantities such as the dipolar matrix and the energy eigenvalues of the free Hamiltonian. So far, to our knowledge, only the linear susceptibilities of the KDP are accurately measured [25].

\subsection{Relation between the microscopic and macroscopic models}

In a first step, we find a relation between the linear susceptibility and these quantities.

We recall that the diagonal element $\rho_{n n}$ of the density matrix represents the population in the state associated with the eigenvalue $\mathcal{E}_{n}$. The constants $\rho_{n n}^{(0)}$ represent these populations at equilibrium.

The relation between the linear susceptibility $\chi^{(1)}$ and the dipolar matrix $\boldsymbol{\mu}$ is given in [8]. We extend it to the case where the levels may be degenerate. We obtain it by equating two expressions of the polarization $\mathbf{P}$. We describe the proof in detail in Appendix A.

Hence, we get the following expression:

$$
\chi_{k \ell}^{(1)}\left(\omega_{p}\right)=\frac{\mathcal{N}}{\hbar} \sum_{m n} \frac{\rho_{m m}^{(0)}-\rho_{n n}^{(0)}}{\omega_{n m}-\omega_{p}} \sum_{\substack{1 \leq r \leq d_{m} \\ 1 \leq s \leq d_{n}}} \mu_{(m, r)(n, s)}^{k} \mu_{(n, s)(m, r)}^{\ell},
$$

where $k$ and $\ell$ denote the coordinates of the tensor $\chi^{(1)}$.

We have applied the fact that the equilibrium values of the populations of the levels associated to the same eigenvalue are the same.

As $\chi^{(1)}$ can be measured experimentally, we use equation (5) to determine the dipolar matrix $\boldsymbol{\mu}$.

From now, we shall make the assumption that only the first three eigenvalues of the free Hamiltonian produce noticeable effects. We recall that the existence of three different levels is a necessary condition for having non linear effects. Therefore the sum in equation (5) is restricted to the first three levels. However, the multiplicity of these energy eigenvalues is still to be determined. 
Remark 2.1. It should also be noted that the coefficients of the dipolar matrix involving the same eigenvalue disappear automatically in equation (5) because of the term $\left(\rho_{m m}^{(0)}-\rho_{n n}^{(0)}\right)$. It implies that the experimental results will not be sufficient to determine all coefficients of the dipolar matrix. We will see how to solve this problem later on.

\subsection{Zernicke's approach}

In this section we recall experimental results from [25]. Hopefully, it will give us more information about the dipolar matrix $\boldsymbol{\mu}$.

In [25], Zernicke measures the electric permittivity of the KDP for several wavelengths of the incoming wave. Then, he uses these results to find an expression for $\chi^{(1)}$ as a function of the incoming wave wavelength that fits reasonably the experimental data.

Because of the symmetries of the KDP, the linear susceptibility $\chi^{(1)}$ must have the following form:

$$
\chi^{(1)}=\left(\begin{array}{ccc}
\chi_{\perp}^{(1)} & 0 & 0 \\
0 & \chi_{\perp}^{(1)} & 0 \\
0 & 0 & \chi_{\|}^{(1)}
\end{array}\right),
$$

when the tensor is written in the axis of the crystal, $\perp$ represents the two directions along the $x$ and $y$ axis of the crystal which are identical by symmetry and $\|$ represents the direction $z$ which is the optical axis of the crystal.

Then Zernicke derives a form for $\chi_{\perp}^{(1)}$ and $\chi_{\|}^{(1)}$ that fits experimental results:

$$
\left\{\begin{array}{l}
\chi_{\perp}^{(1)}(\omega)=\frac{A}{1-\frac{\omega^{2}}{\omega_{12}^{2}}}+\frac{B}{1-\frac{\omega^{2}}{\omega_{13}^{2}}} \\
\chi_{\|}^{(1)}(\omega)=\frac{C}{1-\frac{\omega^{2}}{\omega_{12}^{2}}}+\frac{D}{1-\frac{\omega^{2}}{\omega_{23}^{2}}}
\end{array}\right.
$$

where $A, B, C, D, \omega_{12}, \omega_{13}, \omega_{23}$ are fitted to the experimental curves and $\omega$ represents the frequency of the incoming wave.

Remark 2.2. In fact, Zernicke does not ensure that $\omega_{13}=\omega_{12}+\omega_{23}$ as it should. We have used a method similar to the one described in [25] to find values of $A, B, C, D, \omega_{12}, \omega_{13}, \omega_{23}$ satisfying this physical condition.

Hence, we try to find conditions on the dipolar matrix $\boldsymbol{\mu}$ so that the linear susceptibility $\chi^{(1)}$ obtained from $\boldsymbol{\mu}$ by equation (5) has the form given by equation (6).

\subsection{Conditions such that the dipolar matrix $\boldsymbol{\mu}$ has a Zernicke form}

We equate equations (5) and (6), then the following proposition is easily obtained by identification.

Proposition 2.3. In order to satisfy (6), the dipolar matrix has to verify the following conditions:

- several terms vanish:

$$
\left\{\begin{array}{l}
\mu_{(3, q)(2, p)}^{1}=0, \forall 1 \leq p \leq d_{2}, 1 \leq q \leq d_{3}, \\
\mu_{(3, q)(2, p)}^{2}=0, \forall 1 \leq p \leq d_{2}, 1 \leq q \leq d_{3}, \\
\mu_{(3, q)(1, p)}^{3}=0, \forall 1 \leq p \leq d_{1}, 1 \leq q \leq d_{3},
\end{array}\right.
$$


- some elements are fixed:

$$
\begin{aligned}
& \left\{\begin{array}{l}
\sum_{\substack{1 \leq p \leq d_{1} \\
1 \leq q \leq d_{2}}}\left|\mu_{(2, q)(1, p)}^{1}\right|^{2}=\sum_{\substack{1 \leq p \leq d_{1} \\
1 \leq q \leq d_{2}}}\left|\mu_{(2, q)(1, p)}^{2}\right|^{2}=\frac{\hbar \omega_{21} A}{2 \mathcal{N}\left(\rho_{11}^{(0)}-\rho_{22}^{(0)}\right)}, \\
\sum_{\substack{1 \leq p \leq d_{1} \\
1 \leq q \leq d_{3}}}\left|\mu_{(3, q)(1, p)}^{1}\right|^{2}=\sum_{\substack{1 \leq p \leq d_{1} \\
1 \leq q \leq d_{3}}}\left|\mu_{(3, q)(1, p)}^{2}\right|^{2}=\frac{\hbar \omega_{31} B}{2 \mathcal{N}\left(\rho_{11}^{(0)}-\rho_{33}^{(0)}\right)},
\end{array}\right. \\
& \left\{\begin{array}{l}
\sum_{\substack{1 \leq p \leq d_{1} \\
1 \leq q \leq d_{2}}}\left|\mu_{(2, q)(1, p)}^{3}\right|^{2}=\frac{\hbar \omega_{21} C}{2 \mathcal{N}\left(\rho_{11}^{(0)}-\rho_{22}^{(0)}\right)} \\
\sum_{\substack{1 \leq p \leq d_{2} \\
1 \leq q \leq d_{3}}}\left|\mu_{(3, q)(2, p)}^{3}\right|^{2}=\frac{\hbar \omega_{32} D}{2 \mathcal{N}\left(\rho_{22}^{(0)}-\rho_{33}^{(0)}\right)}
\end{array}\right.
\end{aligned}
$$

- Finally we must have

$$
\sum_{\substack{1 \leq p \leq d_{m} \\ 1 \leq q \leq d_{n}}} \mu_{(n, q)(m, p)}^{k} \mu_{(m, p)(n, q)}^{l}=0, \forall k, l, m, n .
$$

Equation (7) can be visualized through a graph as in Figure 1. The first two levels are the only ones related in all directions (which means the corresponding terms of the dipolar matrix are not vanishing in any direction). The circles represent the three distinct energy levels or eigenspaces. The directions $x$ and $y$, which are the same by symmetry, are represented by $\perp$, the direction $z$ by $\|$.

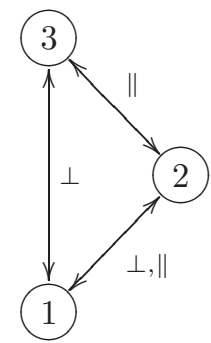

FiguRE 1. Directions of connections between energy levels.

The two equations (8) and (9) relate several elements of the dipolar matrix $\boldsymbol{\mu}$ to the constants given in [25]. Equation (10) does not have as easy an interpretation as the two other points. It comes from the fact that the off-diagonal terms of the linear susceptibility are all vanishing.

It can be easily shown that equation (10) cannot be satisfied if every eigenvalue is of multiplicity one, thus we have an immediate corollary.

Corollary 2.4. At least one of the energy eigenvalues is degenerate.

Remark 2.5. According to [21], this result is not surprising, as symmetry often implies degeneracy.

So far, we have not enough information to determine the dipolar matrix and write a complete model. Thus, we will use the symmetries, which we did not consider until now. 


\section{Model DERIVATiON}

As we have seen, though our model gets more and more precise, it is still far from being complete. We know that at least one of the three eigenvalue is degenerate and the directions of connections between levels are determined.

In this section, we describe the symmetries of the KDP more precisely and study all their implications on the dipolar matrix. The idea is to study the influence of the symmetries on the form of the dipolar matrix and see if it implies that some more coefficients are vanishing. We introduce the theory of linear representations, as it is usual in the study of symmetries in quantum mechanics.

\subsection{KDP symmetries}

The group of symmetry of the KDP is well known, and is described in [1] for instance. This group is classically referred as $\overline{4} 2 m$ (or $D_{2 d}$ ) which we abbreviate by $G$ now. This group has 8 elements and is generated by two symmetries with respect to two orthogonal axes and a third symmetry with respect to one of the mediator planes. The group $G$ does not contain the inversion $\mathbf{r} \mapsto-\mathbf{r}$ (otherwise the crystal would be centrosymmetric and the quadratic nonlinearities would vanish). The full description of the elements of $G$ is given in $[1,6]$.

Next, we give an analytical expression of the elements of the group, according to Table 1.

TABLE 1. Analytical expression of the group $\overline{4} 2 m$.

$$
\begin{aligned}
& \mathrm{Id}:(X, Y, Z) \mapsto(X, Y, Z) \\
& \rho_{X}:(X, Y, Z) \mapsto(X,-Y,-Z) \\
& \rho_{Y}:(X, Y, Z) \mapsto(-X, Y,-Z) \\
& \rho_{Z}:(X, Y, Z) \mapsto(-X,-Y, Z) \\
& \sigma=\sigma_{X+Y}:(X, Y, Z) \mapsto(Y, X, Z) \\
& \tilde{\sigma}=\sigma_{X-Y}:(X, Y, Z) \mapsto(-Y,-X, Z) \\
& \rho_{X} \sigma=\rho_{Y} \tilde{\sigma}:(X, Y, Z) \mapsto(Y,-X,-Z) \\
& \rho_{X} \tilde{\sigma}=\rho_{Y} \sigma:(X, Y, Z) \mapsto(-Y, X,-Z) \\
& \hline
\end{aligned}
$$

We are interested in the dipolar matrix, which involves inner products between eigenfunctions of the free Hamiltonian. We can easily transport the action of the group $G$ to the space of wave functions. Let $\psi$ be any wave function and $g$ an element of the group $G$. Then $g$ acts on $\psi$ by the following equation:

$$
g . \psi(t, \mathbf{r})=\psi\left(t, g^{-1}(\mathbf{r})\right),
$$

where $t$ represents time and $\mathbf{r}$ is the position vector in $\mathbb{R}^{3}$.

Hence, the group $G$ acts on the space of wave functions. Furthermore, if $g$ is a symmetry of the crystal, it commutes with its free Hamiltonian [21]. So the eigenspaces of the free Hamiltonian are stable by the operations of the group $G$. Then we can study separately the action of $G$ on each eigenspace.

The next logical step is to decompose each eigenspace into irreducible linear representations, that is to find a decomposition of this space into a direct sum of the largest, non trivial, subspaces stable by the action of $G$.

As the group $G$ has eight elements, the theory (see page 31 of [22] for instance) states that $G$ has five non isomorphic irreducible representations. Indeed, let us denote by $d_{i}$ the degree of the $i$ th irreducible representation which composes $\mathrm{G}$, we know from [22] that

$$
\sum_{i} d_{i}^{2}=\# G
$$


as we have at least the identity, it is straightforward to see that $G$ has five non isomorphic irreducible representations. These representations are composed of four representations of order one and one of order two, the order being the dimension of the associated subspace.

The irreducible representations are noted I, II, III, IV, V. As the representation V is of degree 2, we denote by $\left(\mathrm{V}_{1}, \mathrm{~V}_{2}\right)$ one of its basis.

We describe the irreducible representations in Table 2.

TABLE 2. Irreducible representations of the KDP. Each column shows the result of the action of the symmetries on a basis of the representation.

\begin{tabular}{|c|c|c|c|c|c|}
\hline Sym. / Rep. & $\begin{array}{c}\mathrm{I} \\
(\mathbf{1}) \\
\end{array}$ & $\begin{array}{c}\text { II } \\
\text { (det) }\end{array}$ & $\begin{array}{l}\text { III } \\
\left(\mathrm{z}^{*}\right) \\
\end{array}$ & $\begin{array}{c}\text { IV } \\
\left(\mathrm{z}^{*} \text { det }\right)\end{array}$ & $\mathrm{V}$ \\
\hline$I$ & 1 & 1 & 1 & 1 & $\left(\begin{array}{ll}1 & 0 \\
0 & 1\end{array}\right)$ \\
\hline$\rho_{X}$ & 1 & 1 & -1 & -1 & $\left(\begin{array}{cc}1 & 0 \\
0 & -1\end{array}\right)$ \\
\hline$\rho_{Y}$ & 1 & 1 & -1 & -1 & $\left(\begin{array}{cc}-1 & 0 \\
0 & 1\end{array}\right)$ \\
\hline$\rho_{Z}$ & 1 & 1 & 1 & 1 & $\left(\begin{array}{cc}-1 & 0 \\
0 & -1\end{array}\right)$ \\
\hline$\sigma_{X+Y}=\sigma$ & 1 & -1 & 1 & -1 & $\left(\begin{array}{ll}0 & 1 \\
1 & 0\end{array}\right)$ \\
\hline$\sigma_{X-Y}=\tilde{\sigma}$ & 1 & -1 & 1 & -1 & $\left(\begin{array}{cc}0 & -1 \\
-1 & 0\end{array}\right)$ \\
\hline$\rho_{X} \sigma=\rho_{Y} \tilde{\sigma}$ & 1 & -1 & -1 & 1 & $\left(\begin{array}{cc}0 & -1 \\
1 & 0\end{array}\right)$ \\
\hline$\rho_{X} \tilde{\sigma}=\rho_{Y} \sigma$ & 1 & -1 & -1 & 1 & $\left(\begin{array}{cc}0 & 1 \\
-1 & 0\end{array}\right)$ \\
\hline
\end{tabular}

Thus, as each eigenspace is stable by $G$, it can be decomposed in a direct sum of irreducible representations. So, in the next section, we will study the form of the dipolar matrix in the case when the eigenspaces are reduced to one irreducible representation.

\subsection{Dipolar coefficients between irreducible representations}

As we have seen, each eigenspace can be decomposed in a direct sum of irreducible representations. So we are interested in calculating dipolar coefficients of the form $\langle\phi, \mathbf{r} \psi\rangle$ where $\psi$ and $\phi$ are basis vectors of two irreducible representations and eigenfunctions of the free Hamiltonian. We must evaluate these inner products, that is integrals of the type $\int \phi^{*} \mathbf{r} \psi$. First, we introduce a projector on the unitary representation.

Definition 3.1. We call $\Pi$ the projector on the identity representation, given by

$$
\Pi \equiv \frac{1}{\# G} \sum_{g \in G} g,
$$

where $\# G$ is the number of elements in $G$. 
TABLE 3. Dipolar coefficients of the KDP.

\begin{tabular}{|c||c|c|c|c|c|c|}
\hline & $\mathrm{I}$ & $\mathrm{II}$ & $\mathrm{III}$ & $\mathrm{IV}$ & $\mathrm{V}_{1}$ & $\mathrm{~V}_{2}$ \\
\hline \hline $\mathrm{I}$ & 0 & 0 & $\alpha_{\mathrm{III}, \mathrm{I}}^{*} \mathbf{e}_{z}$ & 0 & $\alpha_{\mathrm{V}, \mathrm{I}} \mathbf{e}_{x}$ & $\alpha_{\mathrm{V}, \mathrm{I}}^{*} \mathbf{e}_{y}$ \\
\hline $\mathrm{II}$ & 0 & 0 & 0 & $\alpha_{\mathrm{IV}, \mathrm{II}}^{*} \mathbf{e}_{z}$ & $\alpha_{\mathrm{V}, \mathrm{II}} \mathbf{e}_{x}$ & $\alpha_{\mathrm{V}, \mathrm{II}} \mathbf{e}_{y}$ \\
\hline $\mathrm{III}$ & $\alpha_{\mathrm{III}, \mathrm{I}} \mathbf{e}_{z}$ & 0 & 0 & 0 & $\alpha_{\mathrm{V}, \mathrm{III}}^{*} \mathbf{e}_{y}$ & $\alpha_{\mathrm{V}, \mathrm{III}} \mathbf{e}_{x}$ \\
\hline $\mathrm{IV}$ & 0 & $\alpha_{\mathrm{IV}, \mathrm{II}} \mathbf{e}_{z}$ & 0 & 0 & $\alpha_{\mathrm{V}, \mathrm{IV}} \mathbf{e}_{y}$ & $\alpha_{\mathrm{V}, \mathrm{IV}} \mathbf{e}_{x}$ \\
\hline $\mathrm{V}_{1}$ & $\alpha_{\mathrm{V}, \mathrm{I}} \mathbf{e}_{x}$ & $\alpha_{\mathrm{V}, \mathrm{II}} \mathbf{e}_{x}$ & $\alpha_{\mathrm{V}, \mathrm{III}} \mathbf{e}_{y}$ & $\alpha_{\mathrm{V}, \mathrm{IV}} \mathbf{e}_{y}$ & 0 & $\alpha_{\mathrm{V}, \mathrm{V}} \mathbf{e}_{z}$ \\
\hline $\mathrm{V}_{2}$ & $\alpha_{\mathrm{V}, \mathrm{I}} \mathbf{e}_{y}$ & $\alpha_{\mathrm{V}, \mathrm{II}} \mathbf{e}_{y}$ & $\alpha_{\mathrm{V}, \mathrm{III}} \mathbf{e}_{x}$ & $\alpha_{\mathrm{V}, \mathrm{IV}} \mathbf{e}_{x}$ & $\alpha_{\mathrm{V}, \mathrm{V}} \mathbf{e}_{z}$ & 0 \\
\hline
\end{tabular}

We denote by $\boldsymbol{\mu}_{\mathrm{I}, \mathrm{II}}$ the class of dipolar elements computed with one wave function in the irreducible representation I and the other in the irreducible representation II.

As it shown in [6] or [1], a function which vanishes under the action of $\Pi$ must have a vanishing integral (note that this is a sufficient but not a necessary condition to have a vanishing integral). The computation of all elements of the dipolar matrix rises in Table 3 , where $\mathbf{e}_{x}, \mathbf{e}_{y}, \mathbf{e}_{z}$ are the vectors corresponding to the three directions $x, y, z$ and the coefficients $\alpha_{.,}$are complex numbers.

More precisely, we have the following proposition.

Proposition 3.2. The dipolar elements between irreducible representations have the form described in Table 3.

Furthermore, we have $\alpha_{\mathrm{V}, \mathrm{V}} \in \mathbb{R}$.

Proof. To prove this proposition, let $\phi$ and $\psi$ be two eigenfunctions belonging to two irreducible representations. Then we compute $\Pi\left(\phi^{*} \mathbf{r} \psi\right)$, where we recall that $\mathbf{r}$ is the position operator. If $\Pi\left(\phi^{*} \mathbf{r} \psi\right)$ is zero, we are sure that the corresponding element of the dipolar matrix is vanishing. This computation does not depend of the two functions we choose in each representation.

As an example, we compute $\boldsymbol{\mu}_{\mathrm{V}, \mathrm{V}}$.

Let $\left(\phi_{\mathrm{V}_{1}}, \phi_{\mathrm{V}_{2}}\right)$ be a basis for the irreducible representation noted $\mathrm{V}$ of order 2 .

The function $\phi_{\mathrm{V}_{1}}^{*} \mathbf{r} \phi_{\mathrm{V}_{1}}$ is odd, so $\Pi\left(\phi_{\mathrm{V}_{1}}^{*} \mathbf{r} \phi_{\mathrm{V}_{1}}\right)=0$ ( $\Pi$ is the projector on the representation $\mathrm{I}$ that is the unitary representation). Then from [1], we get $\boldsymbol{\mu}_{\mathrm{V}_{1}, \mathrm{~V}_{1}}=0$. We also prove that $\boldsymbol{\mu}_{\mathrm{V}_{2}, \mathrm{~V}_{2}}=0$ in the same way.

We shall now compute $\boldsymbol{\mu}_{\mathrm{V}_{1}, \mathrm{~V}_{2}}$.

$$
\begin{aligned}
\Pi\left(\phi_{\mathrm{V}_{1}}^{*} \mathbf{r} \phi_{\mathrm{V}_{2}}\right) & =\frac{1}{\# G} \sum_{g \in G}\left(\phi_{\mathrm{V}_{1}}^{*} \mathbf{r} \phi_{\mathrm{V}_{2}}\right) \circ g^{-1} \\
& =\frac{1}{\# G} \sum_{g \in G}\left(g \cdot \phi_{\mathrm{V}_{1}}\right)^{*} g \cdot \phi_{\mathrm{V}_{2}}{ }^{t}\left(x^{*} \circ g^{-1}, y^{*} \circ g^{-1}, z^{*} \circ g^{-1}\right),
\end{aligned}
$$

where $x^{*}, y^{*}, z^{*}$ are the projections on each coordinate $\left(x^{*}:\left(x_{1}, x_{2}, x_{3}\right) \mapsto x_{1}, \ldots\right)$.

From Table 2, we know how to calculate the terms $g \cdot \phi_{\mathrm{V}_{1}}, g \cdot \phi_{\mathrm{V}_{2}}$ with $g \in G$ by reading the last column of the table. For instance, $\rho_{Y} \cdot \phi_{\mathrm{V}_{1}}=-\phi_{\mathrm{V}_{1}}$ and $\rho_{X} \sigma \cdot \phi_{\mathrm{V}_{2}}=-\phi_{\mathrm{V}_{1}}$.

The analytical expressions of the elements of $G$ are given in Table 1, and we know the terms $x^{*} \circ g^{-1}, \ldots$

We replace each term in equation (12) and find

$$
\Pi\left(\phi_{\mathrm{V}_{1}}^{*} \mathbf{r} \phi_{\mathrm{V}_{2}}\right)=\frac{1}{8}\left(\begin{array}{c}
0 \\
0 \\
4 z \phi_{\mathrm{V}_{1}}^{*} \phi_{\mathrm{V}_{2}}+4 z \phi_{\mathrm{V}_{2}}^{*} \phi_{\mathrm{V}_{1}}
\end{array}\right) .
$$




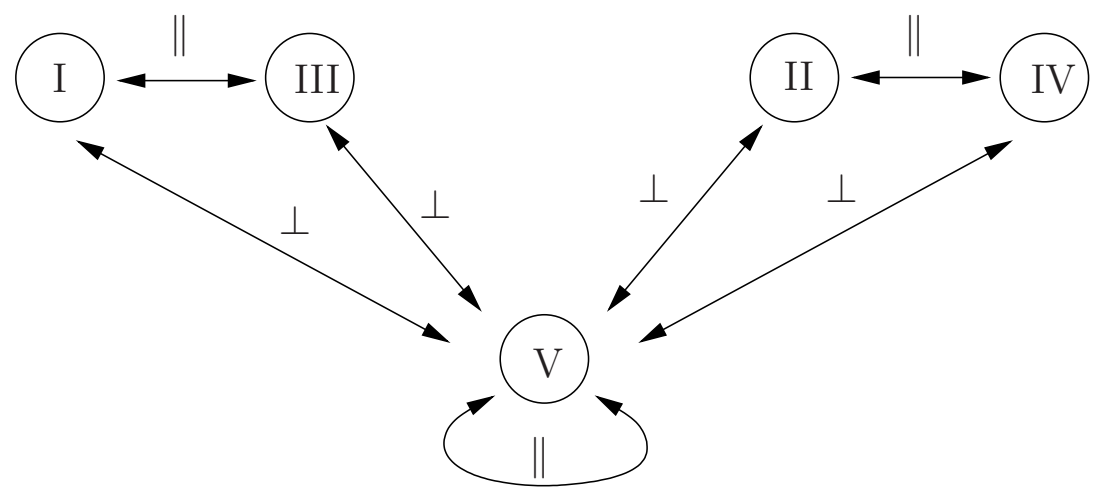

FIGURE 2. Directions of connections between irreducible representations.

However, we have $4 z \phi_{\mathrm{V}_{1}}^{*} \phi_{\mathrm{V}_{2}}+4 z \phi_{\mathrm{V}_{2}}^{*} \phi_{\mathrm{V}_{1}}=8 \operatorname{Re}\left(z \phi_{\mathrm{V}_{1}}^{*} \phi_{\mathrm{V}_{2}}\right)$, so finally

$$
\Pi\left(\phi_{\mathrm{V}_{1}}^{*} \mathbf{r} \phi_{\mathrm{V}_{2}}\right)=\left(\begin{array}{c}
0 \\
0 \\
\operatorname{Re}\left(z \phi_{\mathrm{V}_{1}}^{*} \phi_{\mathrm{V}_{2}}\right)
\end{array}\right) \in \mathbb{R}^{3},
$$

and $\operatorname{Re}\left(z \phi_{\mathrm{V}_{1}}^{*} \phi_{\mathrm{V}_{2}}\right)$ is not necessarily vanishing.

Using the same method for all representations, we get the proposition.

Now we try to translate this proposition into physical conditions. We can visualize the directions of connections between irreducible representations by the graph depicted in Figure 2. Each circle represents an irreducible representation. An arrow means that the corresponding element of the dipolar matrix $\boldsymbol{\mu}$ may be non vanishing. Again, it could vanish for some other reason, because we have derived sufficient but not necessary conditions for an element to vanish.

\subsection{Development of fundamental model}

As it was previously stated, we know that most of the elements of the dipolar matrix are vanishing.

We construct models by taking a decomposition in irreducible representations of each of its three eigenspaces. It follows immediately from Figure 2 that the number of models is not bounded. Our purpose is to find the fundamental model, that is a model with the least number of states i.e. the sum of multiplicities of the three eigenvalues has to be the smallest possible. Since we know that at least one eigenvalue is degenerate, the smallest model has at least four states.

We can prove the following proposition (see Appendix C for details).

Proposition 3.3. The fundamental model has 5 states: its first level is three-fold degenerate.

We can give an example of such a model. See Figure 3 for its decomposition in irreducible representations. We shall call it the fundamental model. Each level represents an eigenspace. Each square represents an irreducible representation as listed in Table 2. We have divided the representation $\mathrm{V}$ in two squares as it is of degree 2. Note that for aesthetic reasons, we have not drawn all the connections. 
Level 3

Level 2

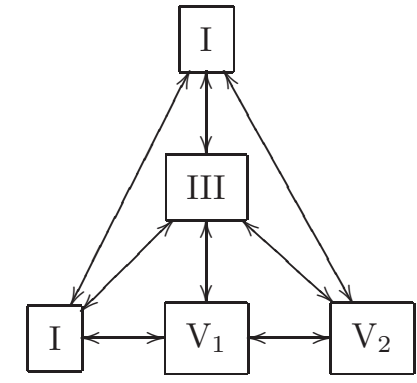

FiguRE 3. Fundamental model.

\section{MODEL ANALYSIS}

In this section, we study the fundamental model we have defined in the previous section.

We recall that we want to solve the Maxwell-Bloch set of equations for the crystal. Apart from the dipolar matrix $\boldsymbol{\mu}$, we have all the information we need. The density matrix $\rho$ is given by the Bloch equations (3). Then we can compute the polarization term $\mathbf{P}$ which is a function of $\rho$ as shown in (4). Finally the polarization is substituted into the Maxwell equations (1) giving us the electromagnetic field (E, B).

The conditions implied by (7) give us the form of the dipolar matrix in the case of the fundamental model. This can be summarized in Table 4 , where stars $\left(^{*}\right)$ and (?) denote non vanishing coefficients. We recall that the dipolar matrix $\boldsymbol{\mu}$ is Hermitian.

TABLE 4. Form of the dipolar matrix $\boldsymbol{\mu}$ (fundamental model).

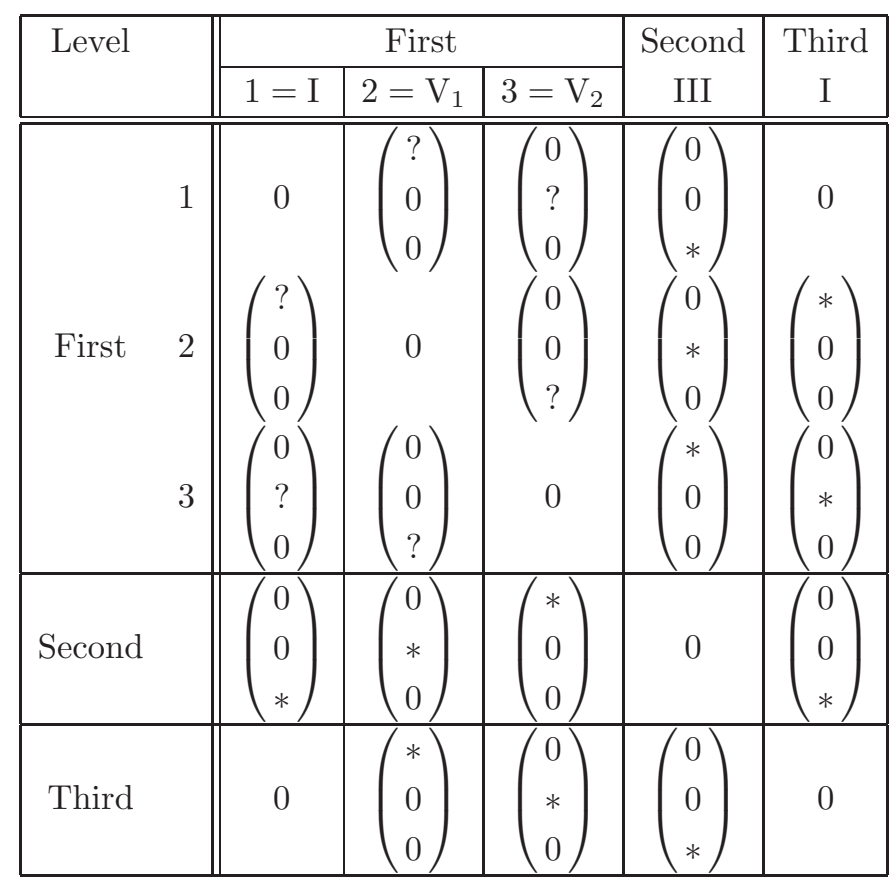


Then we can easily prove the next proposition.

Proposition 4.1. The fundamental model satisfies all the conditions implied by Zernicke's approach (namely equations (7)-(10)). Furthermore, the elements of the dipolar matrix connecting two distinct eigenspaces are fixed in modulus.

Proof. Table 4 implies that system (7) is satisfied by our model. Furthermore, each sum involved in equations (8) and (9) has only one non-vanishing term (from Table 4). For example, we have $\sum_{\substack{1 \leq p \leq d_{1} \\ 1 \leq q \leq d_{2}}}\left|\mu_{(2, q)(1, p)}^{1}\right|^{2}=\left.\left|\mu_{2(1,2)}^{1}\right|\right|^{2}$, since among the dipolar moments involved in this sum, $\boldsymbol{\mu}_{2(1,2)}$ is the only one with a non-vanishing coordinate in the first variable. Hence $\left|\mu_{2(1,2)}^{1}\right|$ is fixed in modulus by the right member of equation (8). Then we proceed with the other sums in (8) and (9) and we deduce the modulus of all the non-vanishing elements of the dipolar moment matrix connecting two distinct eigenspaces.

However, the phases of all the coefficients and the connections inside the first level (marked by "?" in Table 4) are still to be determined.

Concerning the phases, with a convenient choice of the eigenvectors of the free Hamiltonian, we can show the following result.

Proposition 4.2. All but one coefficients of the dipolar matrix $\boldsymbol{\mu}$ can be assumed real.

Proof. Let $\left(\phi_{1}, \phi_{2}, \phi_{3}, \phi_{4}, \phi_{5}\right)$ be a basis of the free Hamiltonian, ordered as in Figure 3 i.e. $\phi_{1}$ and $\phi_{5}$ are two bases of the irreducible representation I, $\phi_{2}$ and $\phi_{3}$ span the irreducible representation of order 2 and $\phi_{4}$ is a basis of III.

We have seen that the elements of the dipolar matrix $\boldsymbol{\mu}$ do not have more than one non-vanishing coordinate.

We chose to freeze the wave-function $\phi_{5}$, we shall adjust the phases of the other wave-function in order to have the maximum number of elements of the dipolar matrix $\boldsymbol{\mu}$ real.

We chose the phase of $\phi_{4}$ to have $\boldsymbol{\mu}_{23}=\left\langle\phi_{4}, \mathbf{r} \phi_{5}\right\rangle \in \mathbb{R}^{3}$ (this vector has only one non-vanishing coordinate). Then we compute the phase of $\phi_{1}$ so as to $\boldsymbol{\mu}_{2,(1,1)}=\left\langle\phi_{4}, \mathbf{r} \phi_{1}\right\rangle \in \mathbb{R}^{3}$.

We take the phase of $\phi_{2}$ in order to have $\boldsymbol{\mu}_{2,(1,2)}=\left\langle\phi_{4}, \mathbf{r} \phi_{2}\right\rangle \in \mathbb{R}^{3}$. However, from Proposition 3.2, we get $\mu_{2(1,2)}^{2}=\mu_{2(1,3)}^{1}$, and, from Table 4 , we have $\boldsymbol{\mu}_{2(1,3)} \in \mathbb{R}^{3}$.

Finally, we chose the phase of $\phi_{3}$ to have $\boldsymbol{\mu}_{(1,1)(1,3)}=\left\langle\phi_{1}, \mathbf{r} \phi_{3}\right\rangle \in \mathbb{R}^{3}$. As $\mu_{(1,1)(1,3)}^{2}=\mu_{(1,1)(1,2)}^{1}$, we also get $\boldsymbol{\mu}_{(1,1)(1,2)} \in \mathbb{R}^{3}$. We still have to determine $\boldsymbol{\mu}_{3,(1,2)}, \boldsymbol{\mu}_{3(1,3)}, \boldsymbol{\mu}_{(1,1)(1,2)}, \boldsymbol{\mu}_{(1,1)(1,3)}$ et $\boldsymbol{\mu}_{(1,2)(1,3)}$.

Owing to the symmetries of the KDP, we know that $\mu_{3(1,2)}^{1}=\mu_{3(1,3)}^{2}, \mu_{(1,1)(1,2)}^{1}=\mu_{(1,1)(1,3)}^{2}$ and $\mu_{(1,2)(1,3)}^{3} \in \mathbb{R}$, the other coordinates of these vectors are vanishing.

Furthermore, we know that, by symmetry, we have $\mu_{\mathrm{I}, \mathrm{V}_{1}}^{1}=\mu_{\mathrm{I}, \mathrm{V}_{2}}^{2}$. Hence, to determine completely the dipolar matrix, we still have to determine two real numbers and one phase, that is three real numbers. For that purpose, we use an expression of the quadratic susceptibility obtained by continuing the method exposed in Appendix A.

Thanks to the form of the dipolar matrix (Tab. 4), it can be shown that with the fundamental model, the quadratic susceptibility $\chi^{(2)}$ has the right symmetries (as in [8]), that is only six terms of this third-order tensor are not vanishing, moreover these terms can be defined by three distinct reals. 
Proposition 4.3. In the case of the fundamental model, the quadratic susceptibility, whose expression (see Appendix B) is

$$
\left.\begin{array}{l}
\chi_{i j k}^{(2)}\left(\omega_{p}+\omega_{q} ; \omega_{q}, \omega_{p}\right)=-\frac{\mathcal{N}}{\hbar^{2}} \\
\quad \times \sum_{m n \ell}\left\{\frac{\rho_{m m}^{(0)}-\rho_{\ell \ell}^{(0)}}{\left(\omega_{n m}-\left(\omega_{p}+\omega_{q}\right)\right)\left(\omega_{\ell m}-\omega_{p}\right)} \sum_{\substack{1 \leq r \leq d_{l} \\
1 \leq s \leq d m \\
1 \leq t \leq d n}} \mu_{(m, s)(n, t)}^{i} \mu_{(n, t)(\ell, r)}^{j} \mu_{(\ell, r)(m, s)}^{k}\right. \\
-\frac{\rho_{\ell \ell}^{(0)}-\rho_{n n}^{(0)}}{\left(\omega_{n m}-\left(\omega_{p}+\omega_{q}\right)\right)\left(\omega_{n \ell}-\omega_{p}\right)} \sum_{\substack{1 \leq r \leq d_{l} \\
1 \leq s \leq d m \\
1 \leq t \leq d_{n}}} \mu_{(m, s)(n, t)}^{i} \mu_{(\ell, r)(m, s)}^{j} \mu_{(n, t)(\ell, r)}^{k}
\end{array}\right\}
$$

has the following physical symmetries:

$$
\left\{\begin{array}{l}
\chi_{123}^{(2)}=\chi_{213}^{(2)}, \\
\chi_{132}^{(2)}=\chi_{231}^{(2)}, \\
\chi_{312}^{(2)}=\chi_{321}^{(2)} .
\end{array}\right.
$$

Proof. The theory (as shown in page 44 of [8]) states that the non-vanishing terms of the quadratic susceptibility are given in (14). We define

$$
S_{i j k}(\ell, m, n) \equiv\left(\rho_{m m}^{(0)}-\rho_{\ell \ell}^{(0)}\right) \sum_{\substack{1 \leq r \leq d_{\ell} \\ 1 \leq s \leq d m \\ 1 \leq t \leq d_{n}}} \mu_{(m, s)(n, t)}^{i} \mu_{(n, t)(\ell, r)}^{j} \mu_{(\ell, r)(m, s)}^{k} .
$$

It can be shown that thanks to the form of the dipolar matrix (Tab. 4), most of the terms of the sum are vanishing, this is summarized in the following lemma.

Lemma 4.4. For the fundamental model, the function $S$ has the following properties.

- If $l=m, S_{i j k}(\ell, m, n)=0, \forall i, j, k$.

- If the energies of two eigenstates among the three states $\ell, m, n$ are identical and non degenerate then $S_{i j k}(\ell, m, n)=0$.

- If $m, n \in\{2,3\}, S_{1 j k}(\ell, m, n)=S_{2 j k}(\ell, m, n)=0, \forall \ell, j, k$. If $\ell, n \in\{2,3\}, S_{i 1 k}(\ell, m, n)=$ $S_{i 2 k}(\ell, m, n)=0, \forall m, i, k$. If $\ell, m \in\{2,3\}, S_{i j 1}(\ell, m, n)=S_{i j 2}(\ell, m, n)=0, \forall m, i, j$.

- If $i=k, S_{i j k}(1, m, 1)=0, \forall m \in\{2,3\}$. If $i=j, S_{i j k}(1,1, n)=0, \forall n \in\{2,3\}$. If $j=k, S_{i j k}(\ell, 1,1)=$ $0, \forall \ell \in\{2,3\}$.

Thanks to this lemma, when estimating the function $S$, we can cancel many triples of the form $(\ell, m, n)$ as listed in the above lemma without further computation. For instance, we compute $\chi_{123}^{(2)}$ for the fundamental model. We have to compute $S_{123}(\ell, m, n)$ for $1 \leq \ell, m, n \leq 3$, which gives 27 terms. Lemma 4.4 removes 18 terms and there remains to compute the terms corresponding to the triples

$$
(1,2,1),(1,3,1),(2,1,1),(2,3,1),(3,1,1) \text { and }(3,2,1) \text {. }
$$

The first triple gives

$$
S_{123}(1,2,1)=\left(\rho_{22}^{(0)}-\rho_{11}^{(0)}\right) \mu_{2(1,3)}^{1} \mu_{(1,3)(1,1)}^{2} \mu_{(1,1) 2}^{3}
$$

which is not necessarily zero. In this expression we have used Table 4 to simplify (15) and to cancel all the terms of the sum but one. 
We proceed with the five other terms of the list (16) using the properties of the dipolar matrix expressed according to Table 4 and Proposition 3.2. Finally we can simplify the complete expression of the quadratic susceptibility (13) in the case of the fundamental model.

For every component of the tensor of quadratic susceptibilities $\chi^{(2)}$, which has 27 entries, we have to compute the sum (13). The sum itself has 27 terms. This is a simple but tedious calculation that can not be reproduced here. Details can be found in [19].

With this computation, it is possible to show that the coefficients (14) are the only non-vanishing coefficients of the quadratic susceptibility $\chi^{(2)}$ in the case of the fundamental model. Furthermore, the relations (14) are also verified by this model.

We still need three real numbers to determine completely the dipolar matrix $\boldsymbol{\mu}$. We fit the missing parameters with the few experimental results, we have found so far (alternatively we could use [18], which relates the linear and quadratic susceptibilities of the KDP) for the quadratic susceptibility given by equation (13). Indeed equation (13) is linear with respect to the unknowns we would like to compute (the elements of the dipolar matrix $\boldsymbol{\mu}$ corresponding to connections between states of the first energy level, which is three-fold degenerate). We can use the same kind of method, we used for the linear susceptibility $\chi^{(1)}$, that is we fix the remaining unknowns in the expression of the quadratic susceptibility to fit experimental results. Moreover, this ensures that the two first orders of the susceptibility given by the fundamental model match experimental results.

Finally, the dipolar matrix $\boldsymbol{\mu}$ is fully determined.

\section{The COMPLETE DiPOLAR MOMENT MATRIX}

Applying the previously exposed methods, we get the whole dipolar moment matrix. We recall that the dipolar moment matrix is real except for one of its component in our model. We use the notations introduced in Figure 3. The dipolar moment matrix has the form

$$
\boldsymbol{\mu}=\left(\begin{array}{ccccc}
0 & \left(\begin{array}{l}
a \\
0 \\
0
\end{array}\right) & \left(\begin{array}{l}
0 \\
a \\
0
\end{array}\right) & \left(\begin{array}{l}
0 \\
0 \\
b
\end{array}\right) & 0 \\
\left(\begin{array}{l}
a \\
0 \\
0
\end{array}\right) & 0 & \left(\begin{array}{l}
0 \\
0 \\
c
\end{array}\right) & \left(\begin{array}{l}
0 \\
d \\
0
\end{array}\right) & \left(\begin{array}{l}
e \\
0 \\
0
\end{array}\right) \\
\left(\begin{array}{l}
0 \\
a \\
0
\end{array}\right) & \left(\begin{array}{l}
0 \\
0 \\
c
\end{array}\right) & 0 & \left(\begin{array}{l}
d \\
0 \\
0
\end{array}\right) & \left(\begin{array}{l}
0 \\
e \\
0
\end{array}\right) \\
\left(\begin{array}{l}
0 \\
0 \\
b
\end{array}\right) & \left(\begin{array}{l}
0 \\
d \\
0
\end{array}\right) & \left(\begin{array}{l}
d \\
0 \\
0
\end{array}\right) & 0 & \left(\begin{array}{l}
0 \\
0 \\
f
\end{array}\right) \\
0 & \left(\begin{array}{l}
e \\
0 \\
0
\end{array}\right) & \left(\begin{array}{l}
0 \\
e \\
0
\end{array}\right) & \left(\begin{array}{l}
0 \\
0 \\
f
\end{array}\right) & 0
\end{array}\right)
$$

where the real numbers $b, c, d, e, f$ and the complex number $a$ have to be determined.

A first set of elements ("*” in Fig. 4, namely $b, d, e$ and $f$ ) is given by the linear susceptibility. 
The following values were computed

$$
\left\{\begin{array}{l}
b=2.82561053429502 \times 10^{-29} \mathrm{Cm} \\
d=5.79683987484668 \times 10^{-29} \mathrm{Cm} \\
e=4.67702893481492 \times 10^{-29} \mathrm{Cm} \\
f=4.62614943821704 \times 10^{-29} \mathrm{Cm} .
\end{array}\right.
$$

The missing elements ("?" in Fig. 4, $a$ and $c$ ) are computed to fit the quadratic susceptibility. According to Proposition 4.2, there is one phase to determine for the coefficient $a$. We choose to take $a$ as a real number and adjust its sign to fit experimental data:

$$
\left\{\begin{array}{l}
a=-0.215955642 \times 10^{-27} \mathrm{Cm}, \\
c=0.106065610 \times 10^{-27} \mathrm{Cm} .
\end{array}\right.
$$

Now that the dipolar matrix $\boldsymbol{\mu}$ is determined, we can solve the equations

$$
\left\{\begin{aligned}
\partial_{t} \rho_{j k} & =-i \omega_{j k} \rho_{j k}+\frac{i}{\hbar}[\boldsymbol{\mu} . \mathbf{E}, \rho]_{j k}, \text { for all } 1 \leq j, k \leq 5 \\
\mathbf{P} & =\mathcal{N} \operatorname{tr}(\boldsymbol{\mu} \rho), \\
\partial_{t} \mathbf{B} & =-\operatorname{rot} \mathbf{E} \\
\partial_{t} \mathbf{E} & =\frac{1}{\mu_{0}} \varepsilon^{-1} \operatorname{rot} \mathbf{B}-\boldsymbol{\varepsilon}^{-1} \partial_{t} \mathbf{P} .
\end{aligned}\right.
$$

Hence the Maxwell-Bloch set of equations is closed.

For instance, given the electric field $\mathbf{E}$, we can solve the Bloch equations (3). Then we can compute the polarization through equation (4) and finally inject the computed polarization into the Maxwell equations (1).

\section{A NUMERICAL EXPERIMENT}

We have fully determined the dipolar moment matrix. We can proceed with numerical computations. The plane wave propagates in the axes $(\mathbf{x}, \mathbf{y}, \mathbf{z})$, where $\mathbf{z}$ is its direction of propagation. We consider that the wave-field depends only on one space variable along the propagation axis $\mathbf{z}$.

The discretization of the model is described in [20]. It extends the Maxwell-Bloch discretization in isotropic media ([5] for instance). The Maxwell system is solved by a FDTD method using the Yee scheme. For the Bloch equations, we use a Strang splitting method, the free-Hamiltonian and wave-Hamiltonian part are solved separately. The time derivative of the polarization term involved in the Bloch equations is computed through the Bloch equations. Thus we obtain a scheme of order 2 in space and time.

We use the values of the dipolar moment matrix, we have found in the previous section. The crystal is rotated and cut so as to its optical axis angle with the direction of propagation of the wave is the angle satisfying the phase matching condition and that the incidence of the wave is normal.

The laser source is a Gaussian pulse with a width of $10^{-14} \mathrm{~s}$ and an amplitude of $10^{8} \mathrm{~V} \mathrm{~m}^{-1}$. The wavelength is $1.06 \mu \mathrm{m}$. The wave is polarized along the $y$-axis. The crystal is surrounded with a linear material. In practice the medium parameters are chosen so as to minimize reflexion of the wave at the interfaces with the KDP. The width of the crystal is set to $20 \mu \mathrm{m}$.

In this run, we should observe second harmonic generation. As our model is not restricted to the linear and quadratic susceptibilities, we also expect to see some effects of higher nonlinearities.

First, we show the evolution over time of the second coordinate of the electric field $\mathbf{E}$ in Figure 5 . At time 0 the $\mathbf{E}$-field is chosen parallel to this direction. We see that the amplitude of the field is decreasing as the wave enters the crystal. We will see in Figure 7, how it translates in terms of the frequencies. 


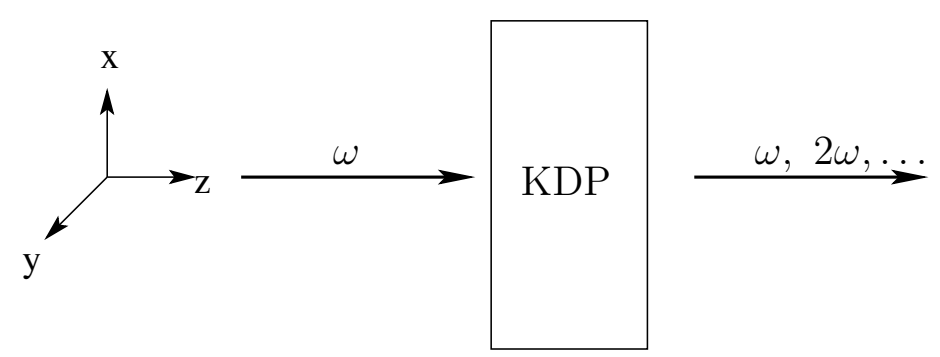

Figure 4. Geometry of the experiment.

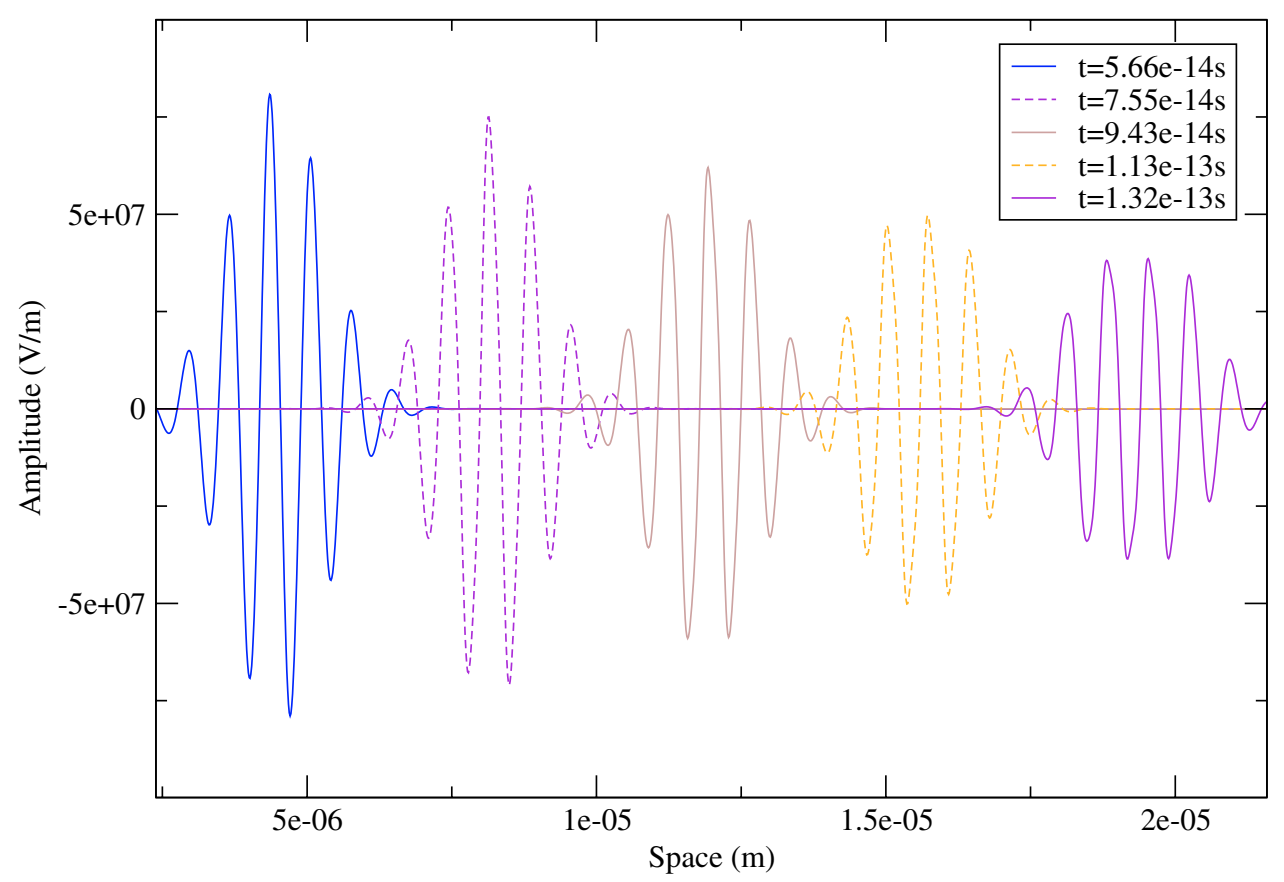

Figure 5. Evolution of the component $E_{y}$ of the electric field $\mathbf{E}$.

Next we show the evolution of $E_{x}$. At the initial time, there is no field along this coordinate as the wave is polarized along $y$. But as the wave is going through the crystal, the amplitude of this component is increasing, as seen in Figure 6, where the electric field $E_{x}$ is drawn at different times. If the phase matching condition is not satisfied, the second harmonic intensity must not increase. This is what is actually observed in our numerical simulations (see [20] for more detail). We have also plotted the theorical amplitude over space of the second harmonic as described in [8] (starting page 78). This theorical amplitude is computed by solving two nonlinear Schrödinger equations for the first two harmonics.

Now, we consider the Fourier transform of the wave field. In the $y$-coordinate (as shown in Fig. 7), the amplitude of the Fourier transform corresponding to the frequency of the incoming wave is decreasing as the wave travels through the crystal. The unit of frequency corresponds to the frequency of the incoming wave. Moreover some third harmonic generation is observed. (Note that second harmonic generation can only be observed on the $x$-component, see below.) 


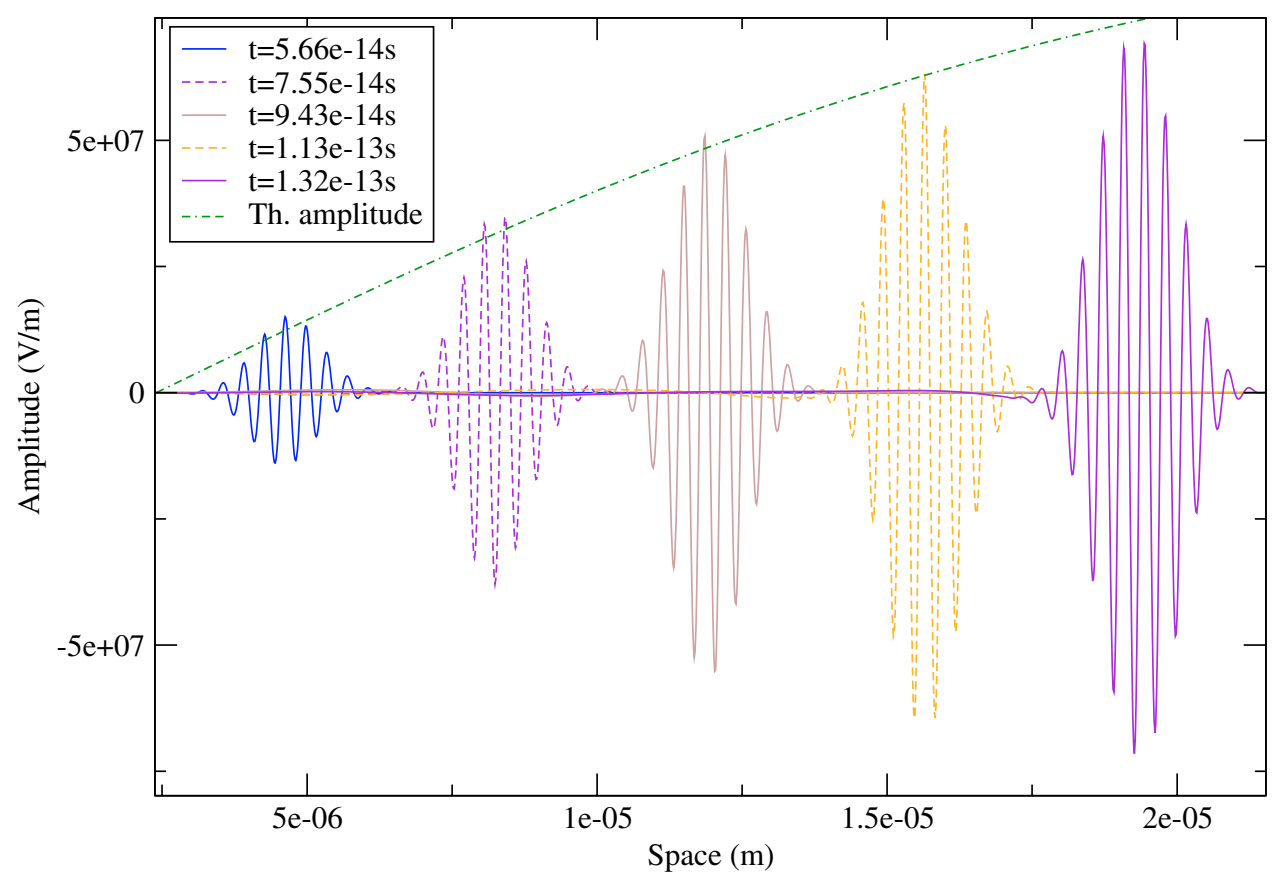

Figure 6 . Evolution of the component $E_{x}$ of the electric field $\mathbf{E}$.

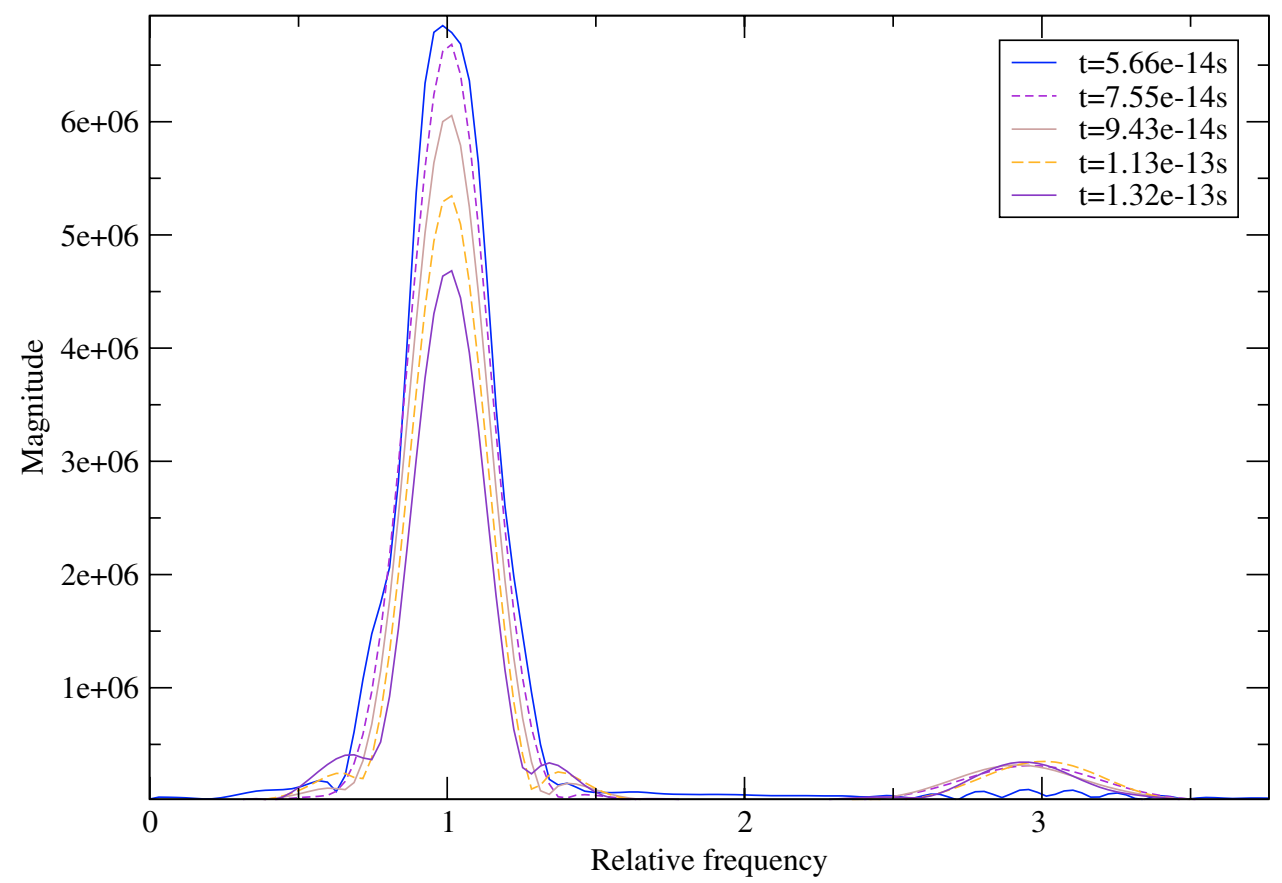

FiguRE 7. Evolution of the Fourier transform of the component $E_{y}$ of the electric field $\mathbf{E}$. 
In Figure 8, we have plotted the Fourier transform of $E_{x}$ for different instants of time. The unit of frequency is the frequency of the incoming wave. The intensity of the second harmonic is actually increasing. Furthermore, we can observe a weak fourth harmonic generation in Figure 8.

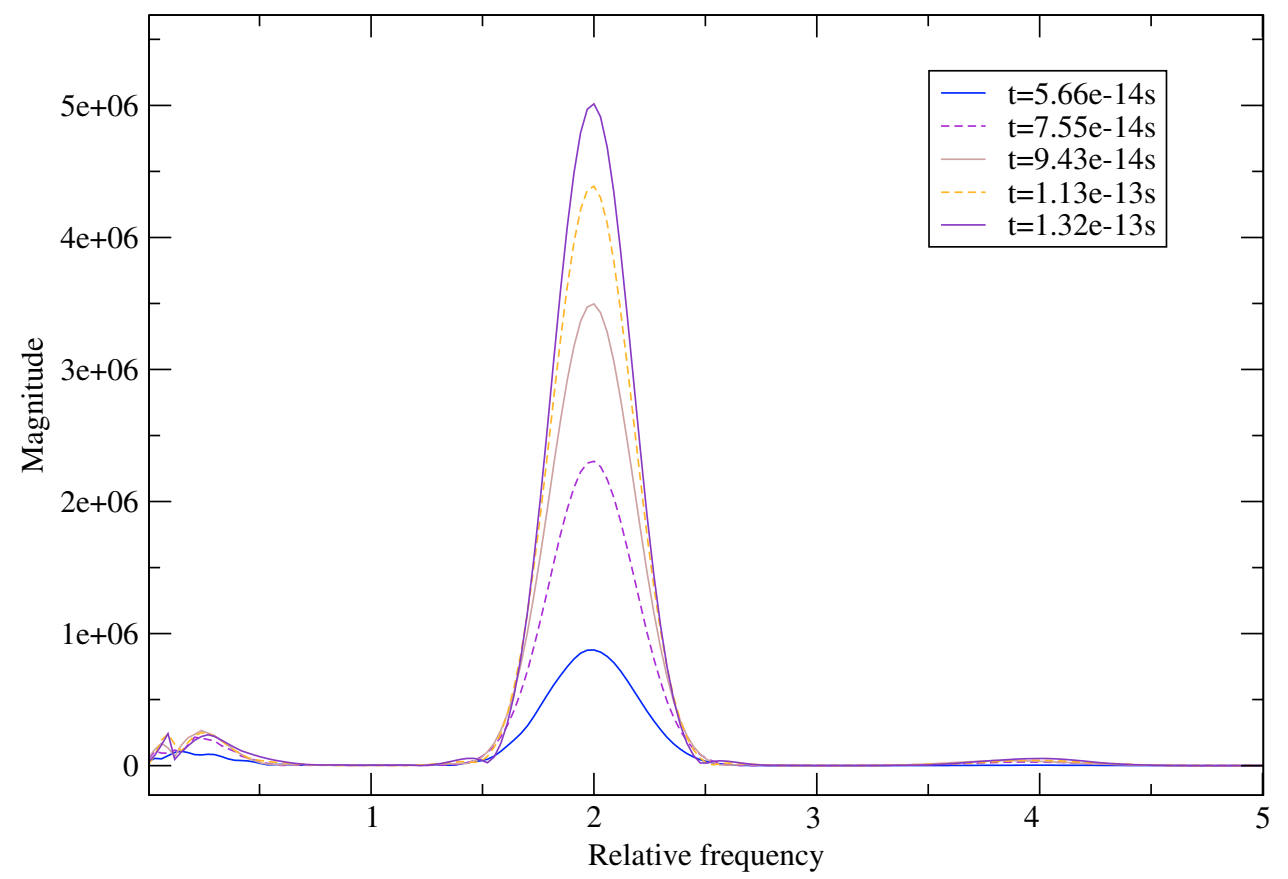

Figure 8. Evolution of the Fourier transform of the component $E_{x}$ of the electric field E.

So far, the numerical results are those expected from the physics and are similar to the results obtained by macroscopic models as in [7] for the first two nonlinear effects. We are then reasonably confident in the validity of our model. But, in our case, we can observe the important effects of the higher nonlinearities as in Figure 7 , where we have a cubic effect. This would not have been observable with macroscopic models such as $[2,3,10,14]$ as they are restricted only to linear and quadratic susceptibilities. [7] renders the third and fourth nonlinearities, but does not take into account the possible saturation of the nonlinearity for instance. We could use our model to improve the accuracy of the dispersion relations used in these models.

\section{Conclusion}

In this paper, we have presented a model for wave-matter interaction in the case of ultrashort laser pulses. It uses the coupled Maxwell-Bloch equations. This model, already studied in detail for isotropic materials, has been seldom used in the case of anisotropic crystals, for which the symmetry group of the crystal has to be taken into account. Additionally, the model requires the knowledge of the dipolar transition matrix, which is not accessible to direct experiments.

To solve this difficulty, we have used the relationships between the susceptibilities, which are easily accessible experimental data [25] and the dipolar matrix, for which we postulate the fundamental form compatible with the symmetries of the crystal. In the present paper we specialized our analysis to the KDP crystal, but the method can be used in full generality.

With this approach, we were able to determine entirely the dipolar matrix of this model of KDP and finally we used this information to perform a numerical simulation. The results were found in good qualitative agreement with those obtained by standard models. 
We believe that these methods can be extended with profit to a large class of materials.

Thanks to the present Maxwell-Bloch model, it is possible to investigate microscopic phenomena involved in wave-matter interaction and to compare this macroscopic description to more standard macroscopic descriptions (e.g. using quadratic nonlinearities). We also believe that our model can be used to validate and improve the macroscopic models. Of course, microscopic model simulations are much more computationally expensive than macroscopic ones and both can be used in a complementary way to achieve an accurate description of the physical phenomena.

\section{Appendix A. Linear susceptibility}

The following derivation of a relation between linear susceptibilities and the dipolar matrix is classical and can be found in [8]. For the reader's convenience, we describe it in this appendix.

We recall the Bloch equations

$$
\partial_{t} \rho_{n m}=-i \omega_{n m} \rho_{n m}-\frac{i}{\hbar}[V, \rho]_{n m}-\gamma\left(\rho_{n m}-\rho_{n m}^{e}\right),
$$

where $\gamma>0$ is a relaxation coefficient, we take $\rho_{n m}^{e}=0$ if $n \neq m$.

We shall make the dipolar approximation, meaning that the potential $V$ is given by

$$
V=-\boldsymbol{\mu} \cdot \mathbf{E} .
$$

We suppose that the interaction between the light and the medium is weak compared to the free Hamiltonian. We replace $V$ by $\varepsilon V$ in the former equation. Then we develop $\rho=\rho^{(0)}+\varepsilon \rho^{(1)}+\varepsilon^{2} \rho^{(2)}+\ldots$ and we identify equal powers of $\varepsilon$, which leads to the following equations:

$$
\partial_{t} \rho_{n m}^{(k)}=-i \omega_{n m} \rho_{n m}^{(k)}-\frac{i}{\hbar}\left[V, \rho^{(k-1)}\right]_{n m}-\gamma \rho_{n m}^{(k)}, k \geq 1 .
$$

For the first order, we take $\rho_{n m}^{(0)}=\delta_{n m} \rho_{n m}^{e}$.

For $k \geq 1$, we define $\sigma_{n m}^{(k)}$ by

thus

$$
\rho_{n m}^{(k)}(t)=\sigma_{n m}^{(k)}(t) \mathrm{e}^{-\left(\gamma+i \omega_{n m}\right) t},
$$

$$
\partial_{t} \sigma_{n m}^{(k)}=-\mathrm{e}^{\left(i \omega_{n m}+\gamma\right) t} \frac{i}{\hbar}\left[V, \rho^{(k-1)}\right]_{n m} .
$$

Then we substitute the value of $\rho^{(0)}$ in the previous expression for $k=1$

$$
\begin{aligned}
{\left[V, \rho^{(0)}\right]_{n m} } & =\sum_{\ell}\left[V_{n \ell} \rho_{\ell m}^{(0)}-\rho_{n \ell}^{(0)} V_{\ell m}\right] \\
& =-\sum_{\ell}\left[\boldsymbol{\mu}_{n \ell} \rho_{\ell m}^{(0)}-\rho_{n \ell}^{(0)} \boldsymbol{\mu}_{\ell m}\right] . \mathbf{E} \\
& =-\left(\rho_{m m}^{(0)}-\rho_{n n}^{(0)}\right) \boldsymbol{\mu}_{n m} . \mathbf{E} .
\end{aligned}
$$

Hence $\sigma^{(1)}$ is given by

$$
\partial_{t} \sigma_{n m}^{(1)}=\frac{i}{\hbar} \mathrm{e}^{\left(i \omega_{n m}+\gamma\right) t}\left(\rho_{m m}^{(0)}-\rho_{n n}^{(0)}\right) \boldsymbol{\mu}_{n m} . \mathbf{E} .
$$

Furthermore, we make the assumption that when $t=-\infty$, the higher order terms of the density matrix vanish: $\rho^{(k)}(t=-\infty)=0, k \geq 1$. Therefore

$$
\lim _{t \rightarrow-\infty} \sigma_{n m}^{(1)}(t)=\lim _{t \rightarrow-\infty} \rho_{n m}^{(1)}(t) \mathrm{e}^{\left(\gamma+i \omega_{n m}\right) t}=0 .
$$


We then have

$$
\sigma_{n m}^{(1)}(t)=\frac{i}{\hbar}\left(\rho_{m m}^{(0)}-\rho_{n n}^{(0)}\right) \int_{-\infty}^{t} \mathrm{e}^{\left(i \omega_{n m}+\gamma\right) s} \boldsymbol{\mu}_{n m} \cdot \mathbf{E}(s) \mathrm{d} s .
$$

We develop $\mathbf{E}$ in Fourier modes

$$
\mathbf{E}(t)=\sum_{p} \mathcal{E}\left(\omega_{p}\right) \mathrm{e}^{-i \omega_{p} t}
$$

and replace $\mathbf{E}$ in (20):

$$
\begin{aligned}
\sigma_{n m}^{(1)}(t) & =\frac{i}{\hbar}\left(\rho_{m m}^{(0)}-\rho_{n n}^{(0)}\right) \int_{-\infty}^{t} \mathrm{e}^{\left(i \omega_{n m}+\gamma\right) s} \boldsymbol{\mu}_{n m} \cdot \sum_{p} \mathcal{E}\left(\omega_{p}\right) \mathrm{e}^{-i \omega_{p} s} \mathrm{~d} s \\
& =\frac{i}{\hbar}\left(\rho_{m m}^{(0)}-\rho_{n n}^{(0)}\right) \boldsymbol{\mu}_{n m} \cdot \sum_{p} \mathcal{E}\left(\omega_{p}\right) \int_{-\infty}^{t} \mathrm{e}^{\left(i\left(\omega_{n m}-\omega_{p}\right)+\gamma\right) s} \mathrm{~d} s \\
& =\frac{i}{\hbar}\left(\rho_{m m}^{(0)}-\rho_{n n}^{(0)}\right) \boldsymbol{\mu}_{n m} \cdot \sum_{p} \mathcal{E}\left(\omega_{p}\right)\left[\frac{\mathrm{e}^{\left(\gamma+i\left(\omega_{n m}-\omega_{p}\right)\right) s}}{\gamma+i\left(\omega_{n m}-\omega_{p}\right)}\right]_{-\infty}^{t} \\
& =\frac{i}{\hbar}\left(\rho_{m m}^{(0)}-\rho_{n n}^{(0)}\right) \boldsymbol{\mu}_{n m} \cdot \sum_{p} \mathcal{E}\left(\omega_{p}\right)\left[\frac{\mathrm{e}^{\left(\gamma+i\left(\omega_{n m}-\omega_{p}\right)\right) t}}{\gamma+i\left(\omega_{n m}-\omega_{p}\right)}\right] .
\end{aligned}
$$

Since $\sigma_{n m}^{(1)}(t)=\rho_{n m}^{(1)}(t) \mathrm{e}^{\left(\gamma+i \omega_{n m}\right) t}$, we have

$$
\rho_{n m}^{(1)}(t)=\frac{i}{\hbar}\left(\rho_{m m}^{(0)}-\rho_{n n}^{(0)}\right) \boldsymbol{\mu}_{n m} \cdot \sum_{p} \mathcal{E}\left(\omega_{p}\right)\left[\frac{\mathrm{e}^{-i \omega_{p} t}}{\gamma+i\left(\omega_{n m}-\omega_{p}\right)}\right] .
$$

The linear polarization $\mathbf{P}^{(1)}$ is given by $\mathbf{P}^{(1)}=\mathcal{N} \operatorname{tr}\left(\boldsymbol{\mu} \rho^{(1)}\right)$ :

$$
\begin{aligned}
\mathbf{P}^{(1)}(t) & =\mathcal{N} \operatorname{tr}\left(\boldsymbol{\mu} \rho^{(1)}\right) \\
& =\mathcal{N} \sum_{m n} \rho_{n m}^{(1)} \boldsymbol{\mu}_{m n} \\
& =\mathcal{N} \sum_{m n} \frac{i}{\hbar}\left(\rho_{m m}^{(0)}-\rho_{n n}^{(0)}\right) \boldsymbol{\mu}_{n m} \cdot \sum_{p} \mathcal{E}\left(\omega_{p}\right)\left[\frac{\mathrm{e}^{-i \omega_{p} t}}{\gamma+i\left(\omega_{n m}-\omega_{p}\right)}\right] \boldsymbol{\mu}_{m n} \\
& =\frac{i \mathcal{N}}{\hbar} \sum_{m n}\left(\rho_{m m}^{(0)}-\rho_{n n}^{(0)}\right) \sum_{p} \frac{\boldsymbol{\mu}_{m n} \boldsymbol{\mu}_{n m} \cdot \mathcal{E}\left(\omega_{p}\right)}{\gamma+i\left(\omega_{n m}-\omega_{p}\right)} \mathrm{e}^{-i \omega_{p} t}
\end{aligned}
$$

The previous equation gives the Fourier modes of $\mathbf{P}^{(\mathbf{1})}$, which we shall denote by $\mathcal{P}^{(1)}\left(\omega_{p}\right)$. By definition, we have $\mathcal{P}^{(1)}\left(\omega_{p}\right)=\chi^{(1)}\left(\omega_{p}\right) \otimes \mathcal{E}\left(\omega_{p}\right)$. In scalar terms, this reads

$$
\mathcal{P}_{k}^{(1)}\left(\omega_{p}\right)=\sum_{\ell} \chi_{k \ell}^{(1)}\left(\omega_{p}\right) \mathcal{E}_{\ell}\left(\omega_{p}\right)
$$

By identification, we have

$$
\chi^{(1)}\left(\omega_{p}\right)=\frac{i \mathcal{N}}{\hbar} \sum_{m n}\left(\rho_{m m}^{(0)}-\rho_{n n}^{(0)}\right) \frac{\mu_{m n} \mu_{n m} .}{\gamma+i\left(\omega_{n m}-\omega_{p}\right)}
$$

which yields the following proposition. 
Proposition A.1. The quantum expression of the linear susceptibility is

$$
\chi_{k l}^{(1)}\left(\omega_{p}\right)=\frac{i \mathcal{N}}{\hbar} \sum_{m n}\left(\rho_{m m}^{(0)}-\rho_{n n}^{(0)}\right) \frac{\mu_{m n}^{k} \mu_{n m}^{l}}{\gamma+i\left(\omega_{n m}-\omega_{p}\right)} .
$$

We can write this expression in the case when energy eigenvalues are degenerate. We recall that $d_{j}$ is the multiplicity of the energy eigenvalue $j$.

Proposition A.2. In the degenerate case, a short expression of the linear susceptibility is:

$$
\chi_{k l}^{(1)}\left(\omega_{p}\right)=\frac{i \mathcal{N}}{\hbar} \sum_{m n} \frac{\rho_{m m}^{(0)}-\rho_{n n}^{(0)}}{\gamma+i\left(\omega_{n m}-\omega_{p}\right)} \sum_{\substack{1 \leq r \leq d_{m} \\ 1 \leq s \leq d_{n}}} \mu_{(m, r)(n, s)}^{k} \mu_{(n, s)(m, r)}^{l} .
$$

We have applied the fact that the equilibrium values of the populations of the levels associated to the same eigenvalue are the same.

\section{Appendix B. Quadratic susceptibility}

In order to obtain an expression for the quadratic susceptibility, we use an analogous method to the one used for the linear susceptibility. The expansion of the density matrix $\rho$ in powers of $\varepsilon$ is pushed one step further [8].

For the second order term of the development of the density matrix $\rho$, we get

$$
\rho_{n m}^{(2)}(t)=-\frac{i}{\hbar} \mathrm{e}^{-\left(\gamma_{n m}+i \omega_{n m}\right) t} \int_{-\infty}^{t} \mathrm{e}^{\left(\gamma_{n m}+i \omega_{n m}\right) s}\left[V, \rho^{(1)}\right]_{n m}(s) \mathrm{d} s
$$

where

$$
\left[V, \rho^{(1)}\right]_{n m}=-\sum_{\ell}\left(\boldsymbol{\mu}_{n \ell} \rho_{\ell m}^{(1)}-\rho_{n \ell}^{(1)} \boldsymbol{\mu}_{\ell m}\right) . \mathbf{E}
$$

Then, after an integration of the previous equation, we identify the two expressions of the quadratic polarization:

$$
\mathbf{P}^{(2)}=\mathcal{N} \operatorname{tr}\left(\boldsymbol{\mu} \rho^{(2)}\right)=\mathcal{N} \sum_{m n} \rho_{n m}^{(2)} \boldsymbol{\mu}_{m n}
$$

which yields:

Proposition B.1. The quantum expression of the quadratic susceptibility is

$$
\begin{aligned}
& \chi_{i j k}^{(2)}\left(\omega_{p}+\omega_{q} ; \omega_{q}, \omega_{p}\right)= \\
& \frac{\mathcal{N}}{\hbar^{2}} \times \sum_{m n \ell}\left\{\left(\rho_{m m}^{(0)}-\rho_{\ell \ell}^{(0)}\right) \frac{\mu_{m n}^{i} \mu_{n \ell}^{j} \mu_{\ell m}^{k}}{\left(\gamma_{n m}+i\left(\omega_{n m}-\left(\omega_{p}+\omega_{q}\right)\right)\right)\left(\gamma_{\ell m}+i\left(\omega_{\ell m}-\omega_{p}\right)\right)}\right. \\
& \left.\quad-\left(\rho_{\ell \ell}^{(0)}-\rho_{n n}^{(0)}\right) \frac{\mu_{m n}^{i} \mu_{\ell m}^{j} \mu_{n \ell}^{k}}{\left(\gamma_{n m}+i\left(\omega_{n m}-\left(\omega_{p}+\omega_{q}\right)\right)\right)\left(\gamma_{n \ell}+i\left(\omega_{n \ell}-\omega_{p}\right)\right)}\right\}
\end{aligned}
$$

As in the linear case, we can write this expression in the case of degenerate energy eigenvalues. 
Proposition B.2. In the degenerate case, a short expression for the quadratic susceptibility (written without relaxations) is

$$
\begin{aligned}
& \chi_{i j k}^{(2)}\left(\omega_{p}+\omega_{q} ; \omega_{q}, \omega_{p}\right)=\frac{\mathcal{N}}{\hbar^{2}} \times \sum_{m n \ell}\left\{\frac{\rho_{\ell \ell}^{(0)}-\rho_{m m}^{(0)}}{\left(\omega_{n m}-\omega_{p}-\omega_{q}\right)\left(\omega_{\ell m}-\omega_{p}\right)} \sum_{\substack{1 \leq r \leq d_{\ell} \\
1 \leq s \leq d_{m} \\
1 \leq t \leq d_{n}}} \mu_{(m, s)(n, t)}^{i} \mu_{(n, t)(\ell, r)}^{j} \mu_{(\ell, r)(m, s)}^{k}\right. \\
& \left.+\frac{\rho_{\ell \ell}^{(0)}-\rho_{n n}^{(0)}}{\left(\omega_{n m}-\omega_{p}-\omega_{q}\right)\left(\omega_{n \ell}-\omega_{p}\right)} \sum_{\substack{1 \leq r \leq d_{\ell} \\
1 \leq s \leq d m \\
1 \leq t \leq d_{n}}} \mu_{(m, s)(n, t)}^{i} \mu_{(\ell, r)(m, s)}^{j} \mu_{(n, t)(\ell, r)}^{k}\right\}
\end{aligned}
$$

\section{Appendix C. Model development (Proof of Prop. 3)}

We recall that our purpose is to find the fundamental model, that is a model with the least number of states, that satisfies conditions implied by experimental results (Zernicke's approach) and symmetries of the crystal. For that matter, we develop a hierarchy of models with increasing complexity.

Before starting our development, we state a first lemma, which proves to be useful in our proof.

Lemma C.1. If the first level does not contain the irreducible representation of order 2, $V$, this representation is necessarily contained in the second and the third level.

Proof. Indeed, the representation $\mathrm{V}$ is necessarily in one of the three levels, since it is the only representation which can introduce the direction $\perp$ between levels (see Figs. 1 and 2).

Then the proof uses essentially Figures 1 and 2 describing the connections between energy levels and irreducible representations.

Since a term of a dipolar matrix $\boldsymbol{\mu}$ may be vanishing for other reasons than symmetry, we begin with a definition.

Definition C.2. We say we add constraints on the dipolar matrix $\boldsymbol{\mu}$ if we assume a priori that some of its elements are vanishing whereas this it not implied by the symmetries of the crystal.

However, as we have not found other reasons for a term of the dipolar matrix $\boldsymbol{\mu}$ to vanish, than those implied by the symmetries of the crystal, we shall prefer not to add constraints to our models if possible. Fortunately, we will see that we can find satisfying models without constraints, moreover adding constraints does not give simpler models.

We recall that the degeneracy of the first eigenvalue is noted $d_{1}$.

First we study the case such that the lowest energy level is non degenerate. We get the following proposition.

Proposition C.3. Without adding constraints, the lowest energy level is necessarily degenerate. With constraints, the less complex model such that the lowest energy level is non-degenerate has at least six states.

Proof. To prove this proposition, we study all the possibilities for the first level. We decompose the first eigenspace corresponding to the lowest energy into irreducible representations. We consider that the lowest eigenvalue is not degenerate. The decomposition of the first energy level, then, necessarily contains one and only one irreducible representation of order 1 . The connections between eigenspaces represented by the coefficients of the dipolar moment matrix $\boldsymbol{\mu}$, have to satisfy Figure 1.

To start, we suppose the first level is restricted to the irreducible representation called I. Then the second level must contain representations III and V, because the first two levels are connected in all directions according to Figure 1 and the representation I is only connected in the direction $\|$ with III and in $\perp$ with V as shown in Figure 2. Hence the second level decomposition into irreducible representations must contain representations called III and V. 
As the first level does not contain the representation $\mathrm{V}$, we know from Lemma C.1 that the representation $\mathrm{V}$ is contained in the decomposition of levels two and three. Then because of the two representations $\mathrm{V}$ in these decompositions, the last two levels are automatically connected in the direction $\|$ (see Fig. 2). But, as III and $\mathrm{V}$ are connected in $\perp$ (Fig. 2), we have just introduced an unneeded direction of connection between the last two energy levels. We can only remove it with constraints.

We use the same method to study the case when the lowest eigenspace can be decomposed into the irreducible representation named III. Again, in this case, we have to add constraints to remove an unneeded direction of connection between two eigenspaces. Finally we remark that levels II and IV are connected symmetrically to representations I and III, with the irreducible representation V and are not connected to representations I and II. Hence the results obtained for representations I and III can be applied to representations II and IV. Without constraints, the lowest energy level is necessarily degenerate.

Hence to find a satisfying model, we need to complexify the first eigenspace. We consider the case of a doubly degenerate level. For a second order degeneracy of the first level, we have the result:

Proposition C.4. If the first level is doubly degenerate, there is no model satisfying conditions (7)-(10) without adding constraints. With constraints, a satisfying model has at least seven states.

As we prefer not to add constraints, the following proposition is immediate:

Corollary C.5. For a model without constraints, the lowest eigenvalue is three-fold degenerate.

Hence, we still need to proceed with the complexification of the first level. For the third order degeneracy of the lowest level, we have a first proposition:

Proposition C.6. There is no model without constraints, satisfying conditions (7)-(10), if the first level does not contain the representation $V$. With constraints, such a model has at least seven states.

The sketch of the proof is the same as in the two previous cases.

As we have shown previously, if the decomposition into irreducible representations of the eigenspace corresponding to the lowest eigenvalue contains the representation $\mathrm{V}$ of order 2 , there exists 5 state models satisfying the conditions implied by Zernicke without constraints. As an example we have the model described by Figure 3. This proves Proposition 3.

Remark C.7. Since we are interested in the simplest model (in term of number of states), we do not study the cases when the first eigenvalue is degenerate at least four times. In this case, a model satisfying the conditions implied by Zernicke has at least 6 states.

\section{REFERENCES}

[1] P.W. Atkins and R.S. Friedman, Molecular Quantum Mechanics. Oxford University Press (1996).

[2] H.J. Bakker, P.C.M. Planken, L. Kuipers and A. Lagendijk, Phase modulation in second-order nonlinear-optical processes. Phys. Rev. A 42 (1990) 4085-4101.

[3] H.J. Bakker, P.C.M. Planken and H.G. Muller, Numerical calculation of optical frequency-conversion processes: a new approach. J. Opt. Soc. Am. B 6 (1989) 1665-1672.

[4] B. Bidégaray, A. Bourgeade and D. Reignier, Introducing physical relaxation terms in Bloch equations. J. Comput. Phys. 170 (2001) 603-613.

[5] B. Bidégaray, Time discretizations for Maxwell-Bloch equations. Numer. Methods Partial Differential Equations 19 (2003) 284-300.

[6] D.M. Bishop, Group theory and chemistry. Dover Press (1973).

[7] A. Bourgeade and E. Freysz, Computational modeling of second-harmonic generation by solution of full-wave vector Maxwell equations. J. Opt. Soc. Am. B $\mathbf{1 7}$ (2000) 226-234.

[8] R.W. Boyd, Nonlinear Optics. Academic Press (1992).

[9] M.M. Choy and R.L. Byer, Accurate second-order susceptibility measurements of visible and infrared nonlinear crystals. Phys. Rev. B 14 (1976) 1693-1706. 
[10] T. Ditmire, A.M. Rubenchik, D. Eimerl and M.D. Perry, Effects of cubic nonlinearity on frequency doubling of high-power laser pulses. J. Opt. Soc. Am. B 13 (1996) 649-655.

[11] R. Eckardt, H. Masuda, Y.X. Fan and R.L. Byer, Absolute and relative nonlinear optical coefficients of $\mathrm{KDP}, \mathrm{KDP}^{*}, \mathrm{BaB}_{2} \mathrm{O}_{4}$, $\mathrm{LiIO}_{3}, \mathrm{MgO}: \mathrm{LiNbO}_{3}$ and KTP measured by phase-matched second-harmonic generation. IEEE J. of Quantum Electr. 26 (1990) 922-933.

[12] D. Eimerl, Electro-optic, linear and nonlinear optical properties of KDP and its isomorphs. Ferroelectrics 72 (1987) 95-139.

[13] J. Jerphagnon and S.K. Kurtz, Optical nonlinear susceptibilities: accurate relative values for quartz, ammonium dihydrogen phosphate, and potassium dihydrogen phosphate. Phys. Rev. B 1 (1970) 1739-1744.

[14] N.C. Kothari and X. Carlotti, Transient second-harmonic generation: influence of effective group-velocity dispersion. J. Opt. Soc. Am. B 5 (1988) $756-764$.

[15] S.K. Kurtz, J. Jerphagnon and M.M. Choy, Nonlinear dielectric susceptibilities. Landolt-Boernstein new series 3 (1979) 671743.

[16] B.F. Levine, Bond-charge calculation of nonlinear optical susceptibilities for various crystal structures. Phys. Rev. B 7 (1973) 2600-2626.

[17] R. Maleck Rassoul, A. Ivanov, E. Freysz, A. Ducasse and F. Hache, Second-harmonic generation under phase-velocity and group-velocity mismatch: influence of cascading self-phase and cross-phase modulation. Opt. Lett. 22 (1997) 268-270.

[18] R.C. Miller, Optical harmonic generation in piezoelectric crystals. Appl. Phys. Lett. 5 (1964) 17-19.

[19] O. Saut, Étude numérique des nonlinéarités d'un cristal par résolution des équations de Maxwell-Bloch, Ph.D. Thesis, INSA Toulouse (2003).

[20] O. Saut, Computational modeling of ultrashort powerful laser pulses in an anisotropic crystal. J. Comput. Phys. (2004) (to appear).

[21] L.I. Schiff, Quantum Mechanics. Mc Graw-Hill International Editions (1995).

[22] J.P. Serre, Représentations linéaires des groupes finis. Hermann (1998).

[23] I. Shoji, T. Kondo, A. Kitamoto, M. Shirane and R. Ito, Absolute scale of second-order nonlinear-optical coefficients. J. Opt. Soc. Am. B 14 (1997) 2268-2294.

[24] J.P. Van Der Ziel and N. Bloembergen, Temperature dependence of optical harmonic generation in $\mathrm{KH}_{2} \mathrm{PO}_{4}$ ferroelectrics. Phys. Rev. 135 (1964) 1662-1669.

[25] F. Zernicke, Refractive indices of ADP and KDP between $2000 \AA$ and $1.5 \mu \mathrm{m}$. J. Opt. Soc. Am. 54 (1964) 1215-1220.

[26] R.W. Ziolkowski, J.M. Arnold and D.M. Gogny, Ultrafast pulse interactions with two-level atoms. Phys. Rev. A 52 (1995) 3082-3094.

To access this journal online:

www.edpsciences.org 